Piotr Wójcik

Wyższe Seminarium Duchowne w Drohiczynie

\title{
La clonazione umana nella letteratura bioetica italiana. Il fatto biologico. Lo scopo riproduttivo e terapeutico
}

\section{KLONOWANIE CZŁOWIEKA WE WŁOSKIEJ LITERATURZE BIOETYCZNEJ. FAKT BIOLOGICZNY. CEL REPRODUKCYJNY I TERAPEUTYCZNY}

Sukcesy odniesione na polu klonowania zwierząt skłoniły naukowców do podjęcia prób klonowania człowieka. Doniesienia o wynikach przeprowadzonych eksperymentów wywołały reakcję w wielu krajach, czego wyraz znajdujemy w literaturze. We Włoszech zagadnienie klonowania człowieka spotkało się z żywą dyskusją. Opisywano i komentowano prowadzone eksperymenty. Przedstawiano zjawisko klonowania jako fakt zachodzący $w$ świecie roślin i zwierząt oraz możliwości zastosowani go u ssaków. Wśród dyskutantów można by wyróżnić dwie grupy: pierwszą stanowią zwolennicy klonowania a drugą jej oponenci. I jedni i drudzy przedstawiają swoje argumenty. W grupie zwolenników można wyodrębnić tych, którzy nie akceptują możliwości zastosowania klonowania do celów reprodukcyjnych a jedynie dopuszczają klonowanie terapeutyczne do celów leczniczych. W dyskusji ma swój głos również Kościół katolicki, który stoi na straży ludzkiej godności.

Słowa klucze: klonowanie reprodukcyjne, klonowanie terapeutyczne, embrion, komórki macierzyste embrionalne. 


\section{Introduzione}

La fine del XX e l'inizio del XXI secolo, è un periodo di un dinamico progresso scientifico e tecnologico nel campo di biologia e medicina. Uno dei frutti di tale progresso è costituito da nuove tecnologie biomediche, le quali permettono di analizzare, controllare e guidare i processi responsabili dell'inizio della vita dell'organismo e del suo sviluppo. Tale tecnica è rappresentata dalla clonazione che vide aprirsi delle prospettive nuove con la nascita della pecora Dolly, il primo mammifero clonato da una cellula di un individuo adulto. Questo confermò la capacità di varcare dei limiti nuovi dagli scienziati che dispongono di successi della tecnologia moderna ${ }^{11}$.

Le prove di clonazione di mammiferi adulti, coronate dal successo, nonché le prime prove sperimentali di clonazione umana, divennero in molte nazioni, un punto di partenza per una discussione degli scienziati, politici e di tutte le società, sul tema di ammissibilità di utilizzo di tale tecnica nei confronti di un essere umano. La maggior parte dei rappresentanti del mondo della scienza, si contrappone in modo decisivo alla clonazione riproduttiva dell'uomo. Invece un gruppo importante degli scienziati si dichiara favorevole all'ammissibilità della clonazione terapeutica, fatta per ottenere le cellule staminali, sostenendo a volte, che il trasferimento di un nucleo della cellula somatica in un oocito non porta alla formazione di un embrione, ma soltanto alla formazione di un agglomerato di cellule ${ }^{12}$.

\section{La clonazione}

Etimologicamente la parola "clone" deriva dal greco "klon", che significa germoglio o ramoscello" " "Nel linguaggio comune, il significato attribuito al termine clonazione è, in sostanza, quello di produzione di individui geneticamente identici, a partire da un altro individuo, senza ricorrere all'incontro e fusione di cellule germinali, maschile e femminile" 14 .

Nel ambiente naturale il fenomeno della clonazione è ordinario. Nel mondo degli animali e dei vegetali esistono individui che si

\footnotetext{
11 Cf. M. Wegnez, Clonazioni. L’individuo, le cellule e i geni, Bari 2009, p. 7.

12 Cf. I.L. Weissman, Stem cell-scientific, medical, and political issues, "The New England Journal of Medicine" 346 (2002) 20, p. 1599-1602.

13 G. Russo, Clonazione. Aspetti genetici, in: AA.VV., Enciclopedia di bioetica e sessuologia (a cura di G. Russo), Torino 2004, p. 469.

14 L. Ciccone, Bioetica. Storia, principi, questioni, Milano 2003, p. 144; F. D'Agostino, L. Palazzani, Bioetica. Nozioni fondamentali, Brescia 2007, p. 103.
} 
moltiplicano nel modo asessuale. Il clone per esempio è la popolazione dei parameci, che si duplicano per divisione. Dai mammiferi il processo di monogenesi non esiste. Può succedere che i cloni dei mammiferi si siano formati nella fase iniziale dello sviluppo della divisione di uno embrione in due o più. Ne consegue la formazione di soggetti geneticamente identici ${ }^{15}$.

Grazie allo sviluppo biotecnologico sono state elaborate le tecniche che permettono la clonazione dei mammiferi nell'ambiente di laboratorio.

\section{Il fatto biologico}

Per una migliore comprensione del processo di clonare, verranno descritti, dal punto di vista biologico, i classici metodi di questo fenomeno.

I gemelli monoovulari sono geneticamente identici perché hanno lo stesso DNA. Essi derivano dalla fusione di un unico uovo con un unico spermatozoo. Durante le successive divisioni da un embrione si formano i due originari esseri. Ancora non si conosce tutti i meccanismi di questo processo ${ }^{16}$.

Questo naturale fenomeno ha incoraggiato gli scienziati a intraprendere delle prove di separazione dei singoli blastomeri ${ }^{17}$ per ottenere da ognuno di loro, interi embrioni indipendenti ${ }^{18}$. Questo metodo si chiama "fissione gemellare" o altrimenti definita "embryo-splitting". La divisione a metà degli embrioni si fece per la prima volta sul topo ${ }^{19}$ e poi nello stesso modo con gli embrioni nella fase precoce di sviluppo degli altri animali ${ }^{20}$. Essenza di questo metodo di clonare è lo sfruttamento della totipotenzialità dei blastomeri, cioè della loro capacità di differenziarsi per dare origine a tutti i tipi di tessuto di un organismo, posseduti nella tappa prematura di sviluppo ${ }^{21}$.

15 Cf. P. Vezzoni, Si può clonare un essere umano?, Roma-Bari 2003, p. 43; J. A. Modliński, J. Karasiewicz, Klonowanie ssaków mity i rzeczywistość, [in:] AA.VV., Klonowanie człowieka. Fantazje - zagrożenia - nadzieje, Lublin 1999, p. 24-26.

16 Cf. M. Wegnez, Clonazioni. L'individuo,le cellule e i geni, op. cit., p. 56.

17 Cf. H. Bartel, Embriologia, Warszawa 2007, p. 92-100.

18 Cf. P. Vezzoni, Si può clonare un essere umano?, op. cit., p. 43.

19 Cf. A. K. Tarkowski, Experiments on the Development of Isolated Blastomeres of Mouse Eggs, "Nature” 184 (1959), p. 1286-1287.

20 Cf. J. S. Nicholas, B. V. Hall, Experiments on Developing Rats, "Journal of Experimental Zoology" 90 (1942), p. 441-459.

21 "Tutti i miliardi di cellule delle duecento linee cellulari dell'uomo adulto derivano da un'unica cellula, l'embrione unicellulare o zigote, attraverso una serie 
Altro metodo di clonazione, il più noto, è il trasferimento di nucleo; "nucleo-transfer". E' stato sperimentato con successo da Wilmut e dai suoi collaboratori in Scozia quando è nata la pecora Dolly ${ }^{22}$. Questa tecnica prevede due momenti: "nel primo, la cellula - uovo viene enucleata; nel secondo, la cellula di cui si vuole trasferire il nucleo viene fusa con il suddetto uovo, mediante uno shock elettrico, che serve anche da attivatore della divisione dell'embrione artificiale. Questi, una volta iniziato lo sviluppo in vitro, viene trasferito nelle vie genitali della madre" ${ }^{23}$.

I primi esperimenti di nucleo-transfer sono stati effettuati utilizzando il nucleo di cellule di embrioni allo stadio iniziale quando le cellule sono nella fase della totipotenza ${ }^{24}$. Successivamente si cominciò ad usare il nucleo delle cellule adulte. Il primo mammifero che è stato concepito e nato in questo modo era proprio la pecora Dolly ${ }^{25}$. Gli studiosi hanno strutturato 277 embrioni dai quali è nato soltanto un agnello ${ }^{26}$. Negli anni successivi si è provato ad ottenere i cloni d'altre specie ${ }^{27}$.

Il grande interesse per la tecnica di clonazione somatica trova la sua dimostrazione nel desiderio di conoscere i processi avvenenti nei primi stadi di sviluppo dei mammiferi. Una cosa importante sembra

di innumerevoli divisioni cellulari mitotiche (che assicurano l'identica ripartizione dell'informazione genica nelle cellule figlie) e di modificazioni cellulari. In questo senso la cellula dello zigote è una cellula «totipotente», cioè in grado di dare origine a tutte le cellule dell'organismo e contiene tutte le informazioni genetiche che ogni cellula poi utilizzerà.(...) Il primo stadio è quello dello zigote, che è una sola cellula totipotente, derivante dalla fusione dell'ovocita con lo spermatozoo. Il secondo è quello dei blastomeri: nei precocissimi stadi di sviluppo dell'embrione, lo zigote si divide e moltiplica in due, quattro, otto, sedici cellule, chiamate blastomeri, ognuno dei quali è ancora totipotente, cioè ha la capacità di generare un nuovo embrione per embryo splitting (cioè separazione di una o più cellule dall'embrione stesso) accidentale o intenzionale e possiede la capacità di dare origine a tutti i tipi cellulari dell'organismo umano", G. M. Carbone, Le cellule staminali, Che cosa sono? A cosa servono? E lecito usarle?, Bologna 2005, p. 7-9.

Cf. L. Ciccone, Bioetica, op. cit., p. 146.

M. L. Di Pietro, E. Sgreccia, Procreazione assistita e fecondazione artificiale tra scienza, bioetica e diritti, Brescia 1999, p. 111.

Cf. P. L. Falzoi, La clonazione umana tra scienza, terapia ed etica, [in:] AA.VV., Biotecnologia ed etica: cellule staminali e clonazione umani, (a cura di A. Garcia Gomez), Roma 2008, p. 216.

Cf. M. Wegnez, Clonazioni. L'individuo,le cellule e i geni, 56; R. Lucas Lucas, Bioetica per tutti, Milano 2002, p. 102-103.

Cf. L. Ciccone, Bioetica, op. cit., p. 146.

Cf. A. Dinnyes, A. Szmolenszky, Animal cloning by nuclear transfer: state-ofthe-art and future perspectives, "Acta Biochimica Polonica" 52 (2005) 3, p. 586. 
anche la possibilità di sfruttare la clonazione nella farmacologia e nella medicina.

\section{La clonazione riproduttiva}

Lo scopo essenziale della clonazione riproduttiva è la vocazione alla vita e la conduzione alla nascita del nuovo organismo, possedente il materiale genetico identico all'organismo datore del nucleo cellulare ${ }^{28}$.

Teologia

La clonazione di animali "potrebbe essere utilizzata per la produzione di embrioni con caratteristiche genetiche controllate in gran quantità, animali da usare poi per lavoro, ad esempio, nell'agricoltura o nell'attività agonistica"29.

Il metodo del trapianto dei nuclei consente il conseguimento di animali transgenetici, che potrebbero produrre le biomolecole per la terapia medica (proteine quali, per esempio, l'a-1-antitripsina, i fattori VIII e IX della coagulazione, il collageno e gli anticorpi). Gli animali avuti con questo metodo potrebbero diventare datori di organi per gli uomini, aspettanti il trapianto del cuore, reni o fegati ${ }^{30}$.

Come nota Roberto Colombo nel documento "Il divieto della clonazione nel dibattito internazionale. (Aspetti Scientifici, Etici e Giuridici)" della Pontificia Accademia Pro Vita: "dopo un periodo di sperimentazione sull'animale, che ha consentito non solo di perfezionare la tecnica di clonaggio ma anche di iniziare a conoscere gli effetti che la clonazione provoca sullo sviluppo e la sopravvivenza dell'embrione e del feto" 31 è venuto il tempo di iniziare le prove per la clonazione dell'uomo.

Il raggiungimento di questo scopo esigerebbe di far passare tutte le tappe del procedimento di clonare analogico al caso di clonare degli animali. Questo significa, che dopo la prima fase di sviluppo

28 Cf. R. Colombo, La clonazione umana, [in:] Pontificia Academia pro Vita, Il divieto della clonazione nel dibattito internazionale. (Aspetti Scientifici, Etici e Giuridici), Città del Vaticano 2003, p. 6-7.

29 M. L. Di Pietro, E. Sgreccia, Procreazione assistita e fecondazione artificiale tra scienza, bioetica e diritti, p. 114.

30 Cf. A. M. O. Canavessi, M. M. Franco, R. Rumpf, Animal transgenesis: state of the art and applications, "Journal of Applied Genetics" 48 (2007) 1, p. 50, 5455; L. R. Kass, Madrość oburzenia. Dlaczego powinniśmy zakazać klonowania człowiek, [in:] AA.VV., Klonowanie człowieka. Fantazje-zagrożenia-nadzieje, Lublin 1999, p. 165.

R. Colombo, La clonazione umana, op. cit., p. 6. 
nelle condizioni di laboratorio, l'embrione sarebbe stato trasferito nel grembo della donna, dove avrebbe potuto crescere ${ }^{32}$.

I fautori della clonazione riproduttiva dell'uomo danno l'elenco degli argomenti fondanti il suo uso.

Ciccone dice, che: "una prima serie di motivi ruota attorno alla speranza di riuscire cosi a dare un figlio a coppie radicalmente e inguaribilmente sterili, o di dare un figlio sano a coppie affette da alcune ben precise patologie ereditarie; di migliorare le tecniche della Fivet e le sue percentuali di successo; di procedere alla diagnosi preimpianto a vantaggio dell'embrione originario"33.

Esiste anche la lista conosciuta di L. $\mathrm{Kass}^{34}$, secondo la quale i motivi per richiedere la clonazione potrebbero essere: replicare individui particolarmente dotati in qualche campo comunemente ammirato, da quello scientifico a quello sportivo o estetico; replicare individui sani per evitare il rischio di malattie ereditarie; procurare un figlio ad una coppia sterile; avere un numero significativo di soggetti identici geneticamente; determinare con sicurezza il sesso del figlio.

H. Jonas nel suo libro Tecnica, medicina ed etica. Prassi del principio di responsabilità riporta la lista di Kass e aggiunge "la preparazione di atleti per le Olimpiadi e simili competizioni internazionali, e infine la curiosità umana di vedere come va a finire una simile procedura"35.

\section{La clonazione "terapeutica"}

"Una chiarificazione preliminare si rende necessaria a motivo di un uso improprio e ingannevole, nell'ambito del dibattito pubblico, della pretesa distinzione tra clonazione umana «riproduttiva» e «terapeutica». I termini sono stati introdotti per alludere a due differenti destinazioni d'uso"36.

Come è stato segnato, per produrre animali interi, dopo il trasferimento nucleare l'embrione viene reimpiantato nel grembo della

32 Cf. G. Koss, Klonowanie człowieka. Wybrane zagadnienia etyczno-moralne, "Sosnowieckie Studia Teologiczne" 7 (2007) 7, p. 74-75.

33 L. Ciccone, Bioetica, op. cit., p. 149; R. Winston, The promise of cloning for human medicine, "British Medical Journal" 314 (1997) 7085, p. 913.

34 Cf. L. Kass, New beginning in life, in: M. P. Hamilton, The new genetics and the future of the man, Grand Rapids 1972, p. 14-63; L. Ciccone, Bioetica, op. cit., p. 149; M. L. Di Pietro, E. Sgreccia, Procreazione assistita e fecondazione artificiale tra scienza, bioetica e diritti op. cit.,, p. 116.

H. Jonas, Tecnica ed etica. Prassi del principio di responsabilità, Torino 1997, p. 141.

$36 \quad$ R. Colombo, La clonazione umana, op. cit., p. 6. 
femmina (la clonazione riproduttiva). Nella clonazione "terapeutica", invece, l'embrione non viene reimpiantato. Non esiste la possibilità della nascita di un animale intero.

"In realtà, ogni clonazione di un organismo è riproduttiva, nel senso dell'etimo di «riprodurre»: essa, quando risulta efficace, «produce di nuovo» un organismo della stessa specie allo stadio iniziale del suo sviluppo, quello embrionale" 37 . Questo tipo di clonazione prevede di ottenere, per scopi terapeutici e di ricerca, cellule staminali embrionali ${ }^{38}$. Queste cellule possiedono la capacità di auto-rinnovamento illimitato o prolungato, cioè di riprodursi a lungo senza differenziarsi e sono all'origine di tutte le cellule differenziate e specifiche di un organismo adulto ${ }^{39}$. Per motivi della qualità, gli scienziati creano gli embrioni allo scopo di ottenere delle cellule staminali ${ }^{40}$.

Le cellule staminali possono essere totipotenti, multi o pluripotenti, a seconda dello stadio di sviluppo in cui si trovano ${ }^{41}$. Le cellule totipotenti, come è stato detto prima, "sono le cellule indifferenziate che hanno la capacità di svilupparsi in qualsiasi tipo di cellula embrionale ed extraembrionale; diversi sperimenti su topi, ratti, conigli ed anche sull'uomo, hanno dimostrato che blastomeri isolati di mammifero sono capaci di originare un embrione completo" 42 .

Invece le cellule pluripotenti, sono le cellule della massa cellulare interna dell'embrione ${ }^{43}$. Esse possono dare origine a tutti i tipi cellulari presenti nell'individuo adulto e per questo vengono dette Ibidem.

$38 \quad$ Cf. D. van der Kooy, S. Weiss, Why stem cells?, "Science” 287 (2000) 5457, p. 1439; R. Lucas Lucas, Bioetica per tutti, op. cit., p. 107; G. Koss, Sposoby otrzymywania ludzkich komórek macierzystych. Aspekt moralny, "Sosnowieckie Studia Teologiczne" 7 (2005) 7, p. 182-185.

Cf. Pontificia Academia pro Vita, Dichiarazione sulla produzione e sull'uso scientifico e terapeutico delle cellule staminali embrionali umane, Città del Vaticano 2000, p. 4; G.M. Carbone, Le cellule staminali, op. cit., p. 10. Cf. P. Vezzoni, Si può clonare un essere umano?, op. cit., p. 57.

41 Cf. R. Mckay, Stem cells - hype and hope, "Nature", 406 (2000) 6794, p. 361; P. A. Iacobelli, Bioetica della nascita e della morte. Storia (incompiuta)dell'esistere umano, Roma 2008, p. 211-217.

A. Giuli, Inizio della vita umana individuale. Basi biologiche e implicazioni bioetiche, Roma 2005, p. 211.

43 "Alla quarta divisione cellulare, circa 70 ore dopo la fertilizzazione, l'embrione si compone di 16 blastomeri e prende nome di morula: il processo di segregazione cellulare continua formando dai blastomeri più esterni la massa cellulare esterna e dai blastomeri più interni la massa cellulare interna, detta anche nodo embrionale. Dopo la sesta divisione cellulare, l'embrione si compone di 64 cellule viene detto blastula" G.M. Carbone, Le cellule staminali, op. cit., p. 17. 
"pluripotenti" 44 . Però, se vengono separate le une dalle altre, non hanno più la capacità di generare un altro embrione, come invece fanno i blastomeri. Proprio queste cellule interessano di più gli scienziati, perché esse possono essere considerate "come una risorsa sempre rinnovabile di cellule, tessuti e particolari organi sani, in vista dei trapianti terapeutici, che si rendono necessari per coloro i quali soffrono di varie malattie e diversi disturbi, ad esempio, patologie quali il Parkinson ed il diabete di tipo $1 " 45$.

I ricercatori le prelevano dagli embrioni in fase blastocisti, cioè tra il $5^{\circ}$ e il $14^{\circ}$ giorno della fertilizzazione ${ }^{46}$. Ma isolare e prelevare le staminali dalla massa cellulare significa distruggere l'embrione stesso ${ }^{47}$.

Per la prima volta le cellule staminali embrionali umane sono state preparate nell'anno 1998 da J. A. Thomson e collaboratori ${ }^{48}$. Però queste cellule sono state ottenute dagli embrioni creati in vitro, cioè non durante la clonazione. "Questi risultati hanno scosso il mondo sia scientifico, sia biotecnologico - in particolare medico e farmacologico e non meno il mondo commerciale e massmediale: apparivano grandi le speranze che le applicazioni che ne sarebbero seguite avrebbero aperto nuove e più sicure vie per la terapia di gravi malattie"49.

Luso delle staminali embrionali ha però il suo rischio. Si avverò evidente nella sperimentazione sul topo, che le cellule staminali embrionali se sono immesse in un organismo adulto, diventano

$44 \quad$ Cf. A. Giuli, Inizio della vita umana individuale, op. cit., p. 213.

45 J. W. Jung, Clonazione per nuclear transfer: problemi bioetici, [in:] AA.VV., Biotecnologia ed etica: cellule staminali e clonazione umani, (a cura di A. Garcia Gomez), Roma 2008, p. 251; A. Mazzoni, Staminali - possibilità terapeutiche, rapporti tra scienza ed etica, Bologna 2007, p. 15.

46 Cf. B. Mepham, Bioetyka, Warszawa 2008, p. 147; H. Bartel, Embriologia, op. cit., p. 93.

47 Cf. G.M. Carbone, Le cellule staminali, op. cit., p. 24; J. Karasiewicz, J. A. Modliński, Komórki macierzyste ssaków: potencjalne źródto zróżnicowanych komórek do transplantacji, "Postępy Biologii Komórki" 28 (2001) 3, p. 225-226; G. M. Carbone, Le questioni etiche relative alle staminali, [in:] A. Mazzoni, Staminali possibilità terapeutiche, raporti tra scienza ed etica, Bologna 2007, p. 75.

48 Cf. J. A. Thomson, J. Itskovitz-Eldor, S. S. Shapiro, M. A. Waknitz, J. J. Swiergiel, V. S. Marshall, J. M. Jones, Embryonic stem cell lines derived from human blastocysts, "Science" 282 (1998) 5391, p. 1145-1147.

49 Pontificia Academia pro Vita, Dichiarazione sulla produzione e sull'uso scientifico e terapeutico delle cellule staminali embrionali umane, p. 6; J. S. Odorico, D. S. Kaufman, J. A. Thomson, Multilineage differentiation from human embryonic cell lines, "Stem Cells" 19 (2001) 3, p. 197-198. 
tumorigeniche ${ }^{50}$. Questo succede spesso con le cellule totipotenti. Esse possono moltiplicarsi in modo spontaneo e incontrollato. Giorgio Carbone dice, che "allo stato attuale nessun ricercatore è già in grado di isolare le capacità benefiche di sviluppo cellulare da quelle capacità dannose" ${ }^{51}$. Per limitare questo rischio sarebbe stato necessario preparare linee specializzate di cellule differenziate, a seconda della necessità. Purtroppo, questo non toglie da altri rischi. Rimane sempre la possibilità dell'incompatibilità immunologica ${ }^{52}$. Le cellule staminali trasferite in un altro soggetto, producono antigeni, poiché hanno un patrimonio genetico diverso da chi le riceve ${ }^{53}$. D. Solter e J. Gerhart propongono tre vie di clonazione "terapeutica" per evitare queste conseguenze ${ }^{54}$. Queste sono: 1 . Trasferimento di un nucleo di una cellula di un dato soggetto in un oocita umano enucleato; 2 . Trasferimento di un nucleo di una cellula di un dato soggetto in oocita di altro animale ${ }^{55} ; 3$. Riprogrammazione del nucleo di una cellula di un dato soggetto fondendolo con il citoplasma di una cellula staminale embrionale ${ }^{56}$.

Gli scienziati nelle loro ricerche dimostrano, che non è facile ottenere gli embrioni umani nel metodo di clonazione, tanto più portare all'esterno di loro le linee delle cellule staminali embrionali.

50

Cf. Pontificia Academia pro Vita, Dichiarazione sulla produzione e sull'uso scientifico e terapeutico delle cellule staminali embrionali umane, p. 7.

G.M. Carbone, Le cellule staminali, op. cit., p. 25; C. Holden, Versatile cells against intractable diseases, "Science" 297 (2002) 5581, p. 500-502.

Cf. J. W. Jung, Clonazione per nuclear transfer: problemi bioetici, op. cit., p. 251; M. Wegnez, Clonazioni. L'individuo,le cellule e i geni, op. cit., p. 127.

Cf. Pontificia Academia pro Vita, Dichiarazione sulla produzione e sull'uso scientifico e terapeutico delle cellule staminali embrionali umane, p. 8.

D. Solter, J. Gearhart, Putting stem cells to work, "Science" 283 (1999) 5407, p. 1486-1470; A. Serra, Identità e dignità dell'embrione umano: un chi o una cosa?, [in:] A. Mazzoni, Staminali - possibilità terapeutiche, rapporti tra scienza ed etica, Bologna 2007, p. 37.

Cf. J. Fulka jr., H. Fulka, J. S. John, C. Galli, G. Lazzari, I. Lagutina, J. Fulka, P. Loi, Hybrid human embryos - warranting opportunities to augment embryonic stem cell research, "Trends in Biotechnology" 26 (2008), p. 469-474; R. M. Doerflinger, Stem cell experiments: renewing the attack on human embryos, http:// www.nrlc.org/news; B. Mepham, Bioetyka, op. cit., p. 150.

Cf. President's Council of Bioethics, White Paper: Alternative Sources of Pluripotent Stem Cells, Washington, DC, May 2005 http://www.bioethics.gov/reports/ white_paper/index.html; W. B. Hurlbut, R. P. George, M. Grompe, Seeking Consensus: A clarification and defense of altered nuclear transfer, "Hastings Center Report" 36 (2006) 5, p. 42-50; J. W. Jung, Clonazione per nuclear transfer: problemi bioetici, op. cit., p. 249. 
Woo Suk Hwang ha pubblicato nel 2004 e 2005 nel "Science" articoli, nei quali dimostrava, che lui ed i suoi collaboratori avrebbero ottenuto 11 linee delle cellule staminali embrionali, dagli embrioni creati nel processo della clonazione ${ }^{57}$. Gli effetti di queste ricerche però suscitarono dei dubbi. La speciale commissione ha sentenziato che le indagini pubblicate sono inverosimili ${ }^{58}$.

Questa truffa, non ha fermato gli scienziati nella ricerca e nelle prove di ottenere le cellule staminali embrionali. Nell'anno 2006, tre gruppi di ricercatori degli Stati Uniti e tre dell'Europa e uno della Cina hanno dichiarato la loro prontezza di provare a ottenere le cellule staminali embrionali nel processo della clonazione ${ }^{59}$.

\section{Conclusione}

Le riflessioni sulla clonazione, non possono limitarsi soltanto alle tecniche. Non si può dimenticare, che tutto questo procedimento concerne l'uomo, e la sua identità e la sua dignità. Lanalisi dei motivi che portano a intraprendere le prove di clonazione permette di constatare che l'uomo viene ridotto ad un oggetto che serve per conseguire altri obiettivi e non il suo bene. In base a queste ragioni, la Chiesa Cattolica valuta la clonazione come immorale e lo esprime tra l'altro nell'Istruzione Dignitas personae dove constata che creare degli embrioni con l'intenzione di distruggerli per portare aiuto agli malati è profondamente immorale ${ }^{60}$.

Il progresso della scienza è comprensibile ed è anche giusto, tuttavia non bisogna dimenticare che la scienza non deve progredire al costo della vita di esseri innocenti, persino - come è già stato detto - pur quanto nobili siano gli obiettivi.

Parole chiave: la clonazione riproduttiva, la clonazione terapeutica, l'embrione, le cellule staminali embrionali.

57 Cf. W. S. Hwang, Y. J. Ryu, J. H. Park, E.S. Park, E. G. Lee, J. M. Koo, H. Y. Jeon, B. C. Lee, S. K. Kang, S. J. Kim, C. Ahm, J. H. Hwang, K. Y. Pak, J. B. Cibelli, S. Y. Moon, Evidence of a pluripotent human embryonic stem cell line derived from a cloned blastocyst, "Science" 303 (2004) 5664, p. 1669-1674; W. S. Hwang, (e collaboratori), Patient-specific embryonic stem cells derived from human SCNT blastocysts, "Science", 308 (2005) 5729, p. 1777-1783.

Cf. J.W. Jung, Clonazione per nuclear transfer: problemi bioetici, op. cit., p. 249.

Cf. G. Vogel, Picking up the pieces after Hwang, "Science" 312 (2006) 5773, p. 516-517.

Cf. DP 30. 


\section{Bibliografia:}

1. Bartel H., Embriologia, Warszawa 2007.

2. Canavessi A.M.O. , Franco M.M., Rumpf R., Animal transgenesis: state of the art and applications, "Journal of Applied Genetics" 48 (2007) 1.

3. Carbone G.M., Le cellule staminali, Che cosa sono? A cosa Servono? E lecito usarle?, Bologna 2005.

4. Carbone G.M., Le questioni etiche relative alle staminali, [in:] A. Mazzoni, Staminali - possibilità terapeutiche, raporti tra scienza ed etica, Bologna 2007.

5. Ciccone L., Bioetica. Storia, principi, questioni, Milano 2003.

6. Colombo R., La clonazione umana, [in:] Pontificia Academia pro Vita, Il divieto della clonazione nel dibattito internazionale. (Aspetti Scientifici, Etici e Giuridici), Città del Vaticano 2003.

7. D’Agostino F., Palazzani L., Bioetica. Nozioni fondamentali, Brescia 2007.

8. Dinnyes A., Szmolenszky A., Animal cloning by nuclear transfer: stateof-the-art and future perspectives, "Acta Biochimica Polonica" 52 (2005).

9. Doerflinger R.M., Stem cell experiments: renewing the attack on human embryos, http://www.nrlc.org/news.

10. Falzoi P. L., La clonazione umana tra scienza, terapia ed etica, [in:] AA.VV., Biotecnologia ed etica: cellule staminali e clonazione umani, (a cura di A. Garcia Gomez), Roma 2008.

11. Fulka J. jr., Fulka H., John J. S., Galli C., Lazzari G., Lagutina I., Fulka J., Loi P., Hybrid human embryos - warranting opportunities to augment embryonic stem cell research, "Trends in Biotechnology" 26 (2008).

12. Giuli A., Inizio della vita umana individuale. Basi biologiche e implicazioni bioetiche, Roma 2005.

13. Holden C., Versatile cells against intractable diseases, "Science" 297 (2002) 5581.

14. Hurlbut W.B., George R.P., Grompe M., Seeking Consensus: A clarification and defense of altered nuclear transfer, "Hastings Center Report" 36 (2006).

15. Hwang W. S., (e collaboratori), Patient-specific embryonic stem cells derived from human SCNT blastocysts, "Science" 308 (2005) 5729.

16. Hwang W. S., Ryu Y. J., Park J. H., Park E. S., Lee E. G., Koo J.M., Jeon H. Y., Lee B.C., Kang S.K., Kim S.J., Ahm C., Hwang J.H., Pak K.Y., Cibelli J.B., Moon S.Y., Evidence of a pluripotent human embryonic stem cell line derived from a cloned blastocyst, "Science" 303 (2004) 5664.

17. Iacobelli P.A., Bioetica della nascita e della morte. Storia (incompiuta) dell'esistere umano, Roma 2008.

18. Jonas H., Tecnica ed etica. Prassi del principio di responsabilità, Torino 1997.

19. Karasiewicz J., Modliński J. A., Komórki macierzyste ssaków: potencjalne źódto zróżnicowanych komórek do transplantacji, "Postępy Biologii Komórki" 28 (2001) 3.

20. Kass L., New beginning in life, [in:] M.P. Hamilton, The new genetics and the future of the man, Grand Rapids 1972. 
21. Kass L.R., Madrość oburzenia. Dlaczego powinniśmy zakazać klonowania człowiek, [in:] AA.VV., Klonowanie człowieka. Fantazje - zagrożenia - nadzieje, Lublin 1999.

22. Kooy D. van der, Weiss S., Why stem cells?, "Science" 287 (2000) 5457.

23. Koss G., Klonowanie człowieka. Wybrane zagadnienia etyczno-moralne, "Sosnowieckie Studia Teologiczne" 7 (2007) 7.

24. Koss G., Sposoby otrzymywania ludzkich komórek macierzystych. Aspekt moralny, "Sosnowieckie Studia Teologiczne" 7 (2005) 7, p. 182-185.

Teologia moralna

25. Mazzoni A., Staminali - possibilità terapeutiche, rapporti tra scienza ed etica, Bologna 2007.

26. Mckay R., Stem cells - hype and hope, "Nature", 406 (2000) 6794.

27. Mepham B., Bioetyka, Warszawa 2008.

28. Modliński J. A., Karasiewicz J., Klonowanie ssaków mity i rzeczywistość, in: AA.VV., Klonowanie człowieka. Fantazje-zagrożenia-nadzieje, Lublin 1999.

29. Nicholas J. S., Hall B. V., Experiments on Developing Rats, "Journal of Experimental Zoology" 90 (1942).

30. Odorico J. S., Kaufman D. S., Thomson J. A., Multilineage differentiation from human embryonic cell lines, "Stem Cells" 19 (2001) 3.

31. Pontificia Academia pro Vita, Dichiarazione sulla produzione e sull'uso scientifico e terapeutico delle cellule staminali embrionali umane, Città del Vaticano 2000.

32. President's Council of Bioethics, White Paper: Alternative Sources of Pluripotent Stem Cells, Washington, DC, May 2005, http://www.bioethics. gov/reports/white_paper/index.html (10.11.2014).

33. Russo G., Clonazione. Aspetti genetici, in: AA.VV., Enciclopedia di bioetica e sessuologia (a cura di G. Russo), Torino 2004.

34. Serra A., Identità e dignità dell'embrione umano: un chi o una cosa?, [in:] A. Mazzoni, Staminali - possibilità terapeutiche, rapporti tra scienza ed etica, Bologna 2007.

35. Solter D., Gearhart J., Putting stem cells to work, "Science" 283 (1999) 5407.

36. Tarkowski A.K., Experiments on the Development of Isolated Blastomeres of Mouse Eggs, "Nature" 184 (1959).

37. Thomson J. A., Itskovitz-Eldor J., Shapiro S.S., Waknitz M. A., Swiergiel J. J., Marshall V. S., Jones J. M., Embryonic stem cell lines derived from human blastocysts, "Science" 282 (1998) 5391.

38. Vezzoni P., Si può clonare un essere umano?, Roma - Bari 2003.

39. Vogel G., Picking up the pieces after Hwang, "Science" 312 (2006) 5773.

40. Wegnez M., Clonazioni. L'individuo, le cellule e i geni, Bari 2009.

41. Weissman I. L., Stem cell - scientific, medical, and political issues, "The New England Journal of Medicine" 346 (2002) 20.

42. Winston R., The promise of cloning for human medicine, "British Medical Journal" 314 (1997) 7085. 